\title{
Zero-point entropy in stuffed spin-ice
}

\author{
G. C. LAU ${ }^{1 *}$, R. S. FREITAS ${ }^{2}$, B. G. UELAND², B. D. MUEGGE ${ }^{1}$, E. L. DUNCAN¹, P. SCHIFFER ${ }^{2 \star}$ AND \\ R. J. CAVA ${ }^{1 *}$ \\ ${ }^{1}$ Department of Chemistry, Princeton University, Princeton, New Jersey 08544, USA \\ ${ }^{2}$ Department of Physics and Materials Research Institute, Pennsylvania State University, University Park, Pennsylvania 16802, USA \\ *e-mail: glau@princeton.edu; schiffer@phys.psu.edu; rcava@princeton.edu
}

T: he third law of thermodynamics dictates that the entropy of a system in thermal equilibrium goes to zero as its temperature approaches absolute zero. In ice, however, a 'zero point' or residual entropy can be measured-attributable to a high degeneracy in the energetically preferred positions of hydrogen ions associated with the so-called 'ice rules' ${ }^{1,2}$. Remarkably, the spins in certain magnetic materials with the pyrochlore structure of corner-sharing tetrahedra, called 'spin ice', have an equivalent degeneracy of energetically preferred states, and also have a zero-point entropy $y^{3-7}$. Here, we chemically alter $\mathrm{Ho}_{2} \mathrm{Ti}_{2} \mathrm{O}_{7}$ spin ice by 'stuffing' extra Ho magnetic moments into otherwise non-magnetic $\mathrm{Ti}$ sites surrounding the Ho tetrahedra. The resulting series, $\mathrm{Ho}_{2}\left(\mathrm{Ti}_{2-x} \mathrm{Ho}_{x}\right) \mathrm{O}_{7-x / 2}$, provides a unique opportunity to study the effects of increased connectivity between spins on a frustrated lattice. Surprisingly, the zeropoint entropy per spin measured appears unchanged by these excess spins. The results suggest a chemical approach for studying ice-like frustration and other properties of the broad family of geometrically frustrated magnets based on the pyrochlore structure.

The energy equivalence of different configurations for hydrogen ions in ice is an example of geometrical frustration. In general, geometrical frustration occurs when local interactions between a system's components cannot all be simultaneously satisfied due to their spatial arrangement on a regular lattice, leading to a large degeneracy of low-energy states. Geometrical frustration in magnetic materials ${ }^{8,9}$ has led to a wide range of novel physics, and has received particular attention in the rare-earth pyrochlore compounds. These materials have the generic formula $\mathrm{R}_{2} \mathrm{M}_{2} \mathrm{O}_{7}$, where the $\mathrm{R}^{3+}$ and $\mathrm{M}^{4+}$ cations form equivalent interpenetrating, equally sized sublattices of corner-sharing tetrahedra (as shown in Fig. 1$)^{10}$. These systems display low-temperature behaviour ranging from exotic ordered states ${ }^{1-14}$, to spin liquids, in which the spins are correlated yet continue to fluctuate as $T \rightarrow 0$ (ref. 15), to the spinice state.

Spin-ice behaviour has been observed in pyrochlores where the R site is occupied by either Dy or Ho magnetic cations, and non-magnetic Ti or Sn occupy the equivalent M sublattice $e^{5,6}$. The crystal field environment in this structure is such that each Ho or Dy spin must point along the line connecting the centres of its two tetrahedra (that is, either directly towards or away from the centres of the tetrahedra). Coupled with the dipolar and exchange interactions, this anisotropy results in six energetically equivalent low-energy states for each tetrahedron, in which two spins point into and two spins point out from each tetrahedron, exactly analogous to the 'ice rules' for hydrogen positions in water ice. Neutron scattering and magnetic susceptibility measurements have demonstrated that these systems freeze into a disordered spin state at low temperatures, and that the local spin correlations follow the two-in/two-out structure ${ }^{16-19}$. The statistical mechanics of this system are identical to that of the hydrogen ions in ice, and the measured molar entropy of the spin system (obtained by integrating the measured specific heat) is $S=R[\ln 2-$ $(1 / 2) \ln (3 / 2)]$, where $R$ is the gas constant $t^{4}$. This is less than the $S=R \ln 2$ value expected for an Ising system, by precisely the same amount as for ice, and can be understood as a direct consequence of the 'ice rules' as applied by Pauling ${ }^{2}$.

The tetrahedra on the $\mathrm{R}$ and $\mathrm{M}$ sublattices of the pyrochlore spin-ice materials have sides of the same length, that is, the distance between nearest neighbours within each of the two sublattices is the same. Furthermore, the shortest distance between neighbouring $\mathrm{R}$ and $\mathrm{M}$ sites is also exactly the same as this nearest-neighbour distance within each of the two sublattices. As illustrated in Fig. 1a, this implies that if all of the $\mathrm{R}$ and $\mathrm{M}$ sites were considered together as a single lattice, the combined lattice would consist of side-sharing tetrahedra (equivalent to a face-centred-cubic lattice, which can also lead to geometrical frustration ${ }^{20,21}$ ). The geometry of the two sublattices also implies that if a single non-magnetic $\mathrm{M}$ ion was replaced with a magnetic $\mathrm{R}$ ion, the connectivity of the surrounding $\mathrm{R}$ sites would be drastically changed. Instead of only having six nearest-neighbour spins, such a replacement would cause the adjacent R-site spins to have a seventh nearestneighbour spin. Furthermore, the spins on those adjacent R-site tetrahedra would share more than three nearest neighbours, which would be expected to have a substantial impact on the nature of the frustration.

We have explored the consequences of such an increase in connectivity by replacing a fraction of the non-magnetic $\mathrm{Ti}$ ions with $\mathrm{Ho}$ ions in $\mathrm{Ho}_{2} \mathrm{Ti}_{2} \mathrm{O}_{7}$, effectively stuffing extra spins into the spin-ice system. The resultant materials form the series 

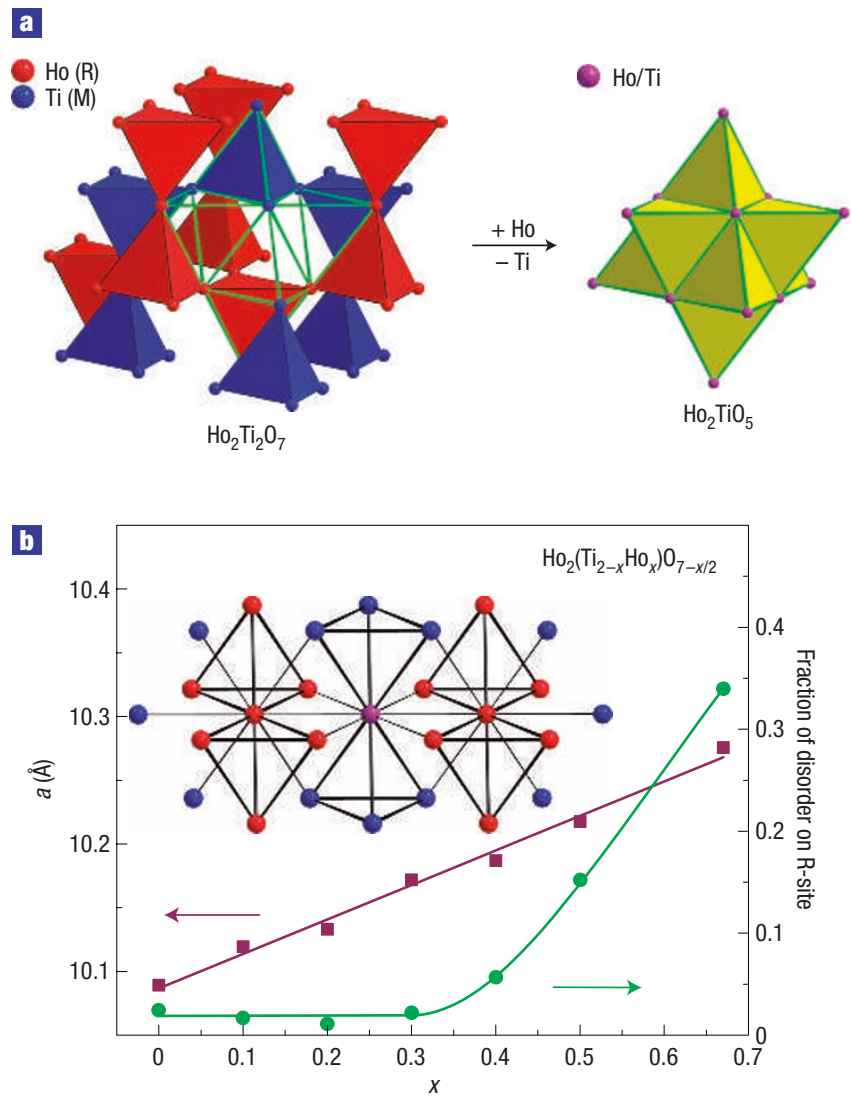

Figure 1 Structural details of the materials studied: $\mathrm{Ho}_{2}\left(\mathrm{Ti}_{2-x} \mathrm{Ho}_{x}\right) \mathbf{0}_{7-x / 2}$. a, The cubic pyrochlore $\left(\mathrm{R}_{2} \mathrm{M}_{2} \mathrm{O}_{7}\right)$ lattice. The corner-sharing tetrahedral sublattices of $\mathrm{H}_{0}$ and $\mathrm{Ti}$ are shown in the $\mathrm{Ho}_{2} \mathrm{Ti}_{2} \mathrm{O}_{7}$ structure (left), with the green outline depicting edge-sharing tetrahedral interactions that develop as extra Ho replace Ti atoms. At $x=0.67$, Ho and Ti reside randomly on the same sites, forming the fluorite phase (right). $\mathbf{b}$, Main panel: lattice parameter and fraction of R-site disorder (occupancy of Ti on the R-site of the pyrochlore structure) in the stuffed material plotted as a function of $x$. The lines are guides to the eye. Standard errors calculated from the best fit to the observed $X$-ray measurements are less than the symbol size. Inset: metal-metal coordination around $\mathrm{Ho}$ (red) and $\mathrm{Ti}$ (blue) in $\mathrm{Ho}_{2} \mathrm{Ti}_{2} \mathrm{O}_{7}$ with only the $\mathrm{R}$ and $M$ sites shown; oxygen atoms are omitted. The bold lines highlight segments of the original corner-sharing tetrahedral motif. The purple atom represents a possible Ti site that can be 'stuffed' with extra Ho.

$\mathrm{Ho}_{2}\left(\mathrm{Ti}_{2-x} \mathrm{Ho}_{x}\right) \mathrm{O}_{7-x / 2}$, where $0 \leq x \leq 0.67$ (synthesis details are given in the Methods section). With the extra Ho, the lattice is expanded (Fig. 1b), and magnetic sites are no longer limited to the vertices of corner-sharing tetrahedra, and can have as many as 12 nearest neighbours (with the average number being $n=6+3 x$ ). Structure refinements revealed that for $0 \leq x \leq 0.3$ Ho replaced Ti strictly on the pyrochlore $\mathrm{M}$-site. Above $x \cong 0.4$, the mixing of Ho and $\mathrm{Ti}$ is no longer restricted to the $\mathrm{M}$ site, and some Ti is situated on the $\mathrm{R}$ site, creating a fraction of disorder in the previously pure Ho corner-sharing sublattice (Fig. 1b). This mixing of ions on the two sites increases with $x$ until Ho and $\mathrm{Ti}$ are completely randomized, and the $\mathrm{R}$ and $\mathrm{M}$ sites become indistinguishable from each other at $x=0.67$ (that is, $\mathrm{Ho}_{2} \mathrm{TiO}_{5}$ ). The $x=0.67$ end member has the fluorite crystal structure-simply a pyrochlore with both $\mathrm{R}$ and $\mathrm{M}$ sites occupied randomly by $\mathrm{Ho}$ and $\mathrm{Ti}$ ions in their stoichiometric ratio $22-24$. The $\mathrm{Ho}$ doping of $\mathrm{Ho}_{2} \mathrm{Ti}_{2} \mathrm{O}_{7}$ thus transforms separate networks of $\mathrm{Ho}$ and $\mathrm{Ti}$ corner-sharing

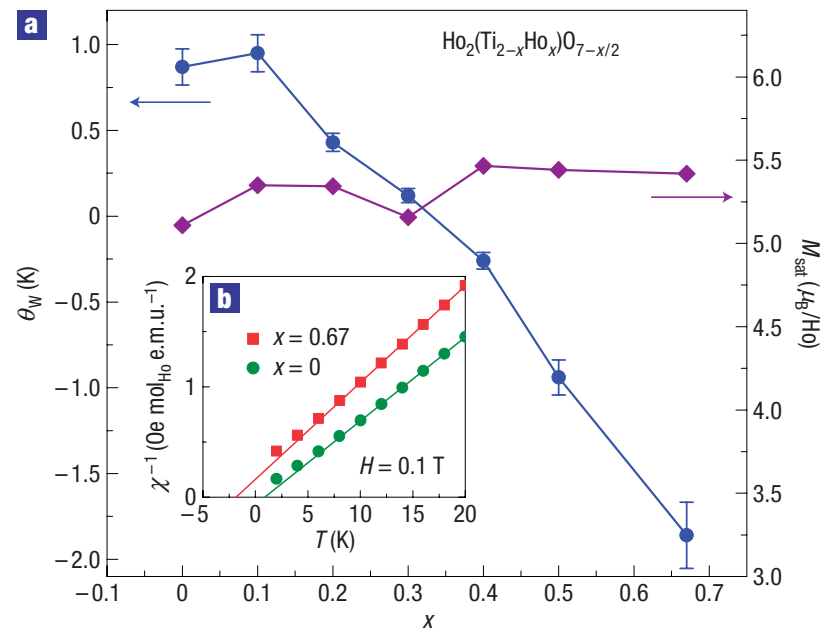

Figure 2 Temperature dependence of the magnetization. a, The Weiss temperature $\left(\theta_{\mathrm{W}}\right)$ and saturation magnetization $(T=2 \mathrm{~K}, H=5.5-7 \mathrm{~T})$ plotted versus $x$ for the various $\mathrm{Ho}_{2}\left(\mathrm{Ti}_{2-x} \mathrm{HO}_{x}\right) \mathrm{O}_{7-x / 2}$ samples. The Weiss temperatures were determined from fits to the Curie-Weiss law on multiple samples for each value of $x$, and the error bars are the computed standard deviations. The uncertainty in the values of $\theta_{\mathrm{W}}$ is estimated from fits to multiple measurements of the temperature-dependent magnetization. The estimated uncertainty in the saturation magnetization is $\pm 5 \%$ on the basis of the uncertainty in sample mass. Note the crossover from positive to negative $\theta_{\mathrm{w}}$ with increasing $x$. b. The inverse d.c. magnetic susceptibility versus temperature for $x=0$ and 0.67 , illustrating the crossover in $\theta_{\mathrm{W}}$ which is taken from the intercept with the horizontal axis (lines are fits to the data used to determine $\theta_{\mathrm{W}}$, as described in the text; the effective moment obtained from these fits is within $2 \%$ of the accepted value for $\mathrm{Ho}$ ).

tetrahedra into a network of edge-sharing tetrahedra, where Ho and $\mathrm{Ti}$ are randomly mixed in a 2:1 ratio.

The stuffing of $\mathrm{Ho}_{2} \mathrm{Ti}_{2} \mathrm{O}_{7}$ offers a unique opportunity to study the nature of ice-like frustration, because the additional Ho ions provide additional connectivity in the magnetic sublattice. In fact, this type of transformation of the connectivity of a frustrated system has not, to our knowledge, been examined previously in any geometrically frustrated magnetic material and would be structurally impossible in water ice. Considering the significant change in topology, it might be expected that the low-temperature spin-ice behaviour would be entirely disrupted. We measured the specific heat, as well as the a.c. and d.c. magnetic susceptibilities of this series, with the intention of investigating how the ice-like properties (for example, the dynamic freezing and the entropy deficit) are altered by enhancing the connectivity.

The initial characterization of the samples came from the d.c. magnetization, which was measured both as a function of temperature and magnetic field. Field-dependent data for all of the samples at $T=2 \mathrm{~K}$ demonstrated that the moment saturated at approximately $5 \mu_{\mathrm{B}} /$ Ho (shown in Fig. $2 \mathrm{a}$ ), which is about half of the theoretically expected value of the full Ho moment in $\mathrm{Ho}_{2} \mathrm{Ti}_{2} \mathrm{O}_{7}$-the factor of one half comes from the projection of the spins in a polycrystalline sample along any given direction ${ }^{25}$. This result is consistent with the Ho ions on both sites being Ising spins with the same total moment of about $10 \mu_{\mathrm{B}} /$ Ho. This is surprising, as the crystal-field environments of the two sublattices should be different. Constant field $M(T)$ data taken for $T=10-20 \mathrm{~K}$ at $H=0.1 \mathrm{~T}$ were fitted to the Curie-Weiss formula to determine the effective moment, $p$, and average mean-field spin-spin interaction as measured through the Weiss temperature, $\theta_{\mathrm{W}}$. We find that $p$ 

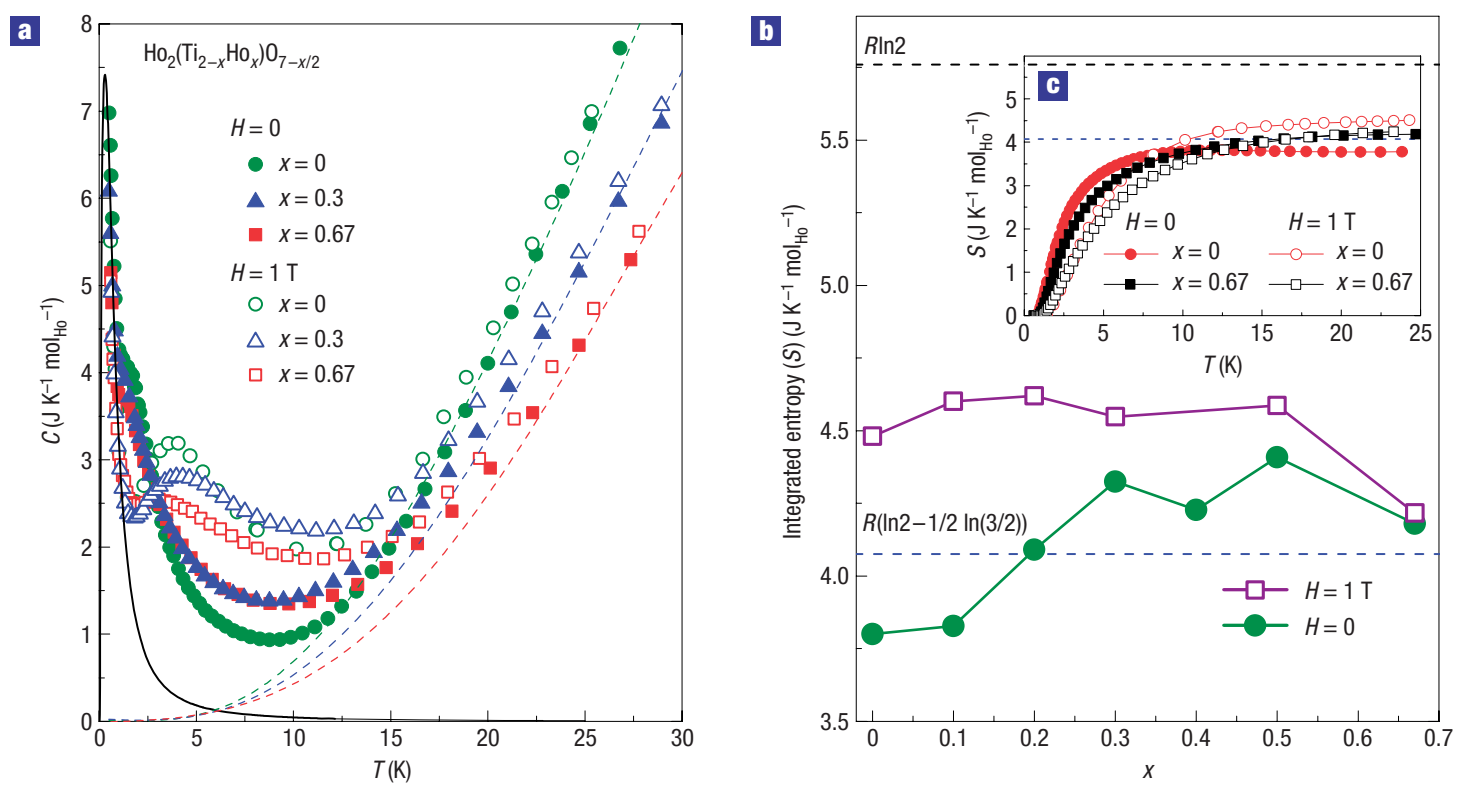

Figure 3 Thermal characterization of stuffed spin-ice. a, The total specific heat of $\mathrm{HO}_{2}\left(\mathrm{Ti}_{2-x} \mathrm{Ho}_{x}\right) \mathrm{O}_{7-x / 2}$ for $x=0,0.3$ and 0.67 at $H=0$ and $1 \mathrm{~T}$. The dashed and solid lines represent the lattice and nuclear contributions, as described in the text. $\mathbf{b}$, The total magnetic entropy (integrated from below $T=1$ to $22 \mathrm{~K}$ ) as a function of $x$ at $H=0$ and $1 \mathrm{~T}$. The blue and black dashed lines represent the predicted ice entropy and total spin entropy values, respectively. c, The temperature dependence of the integrated entropy for our limiting compositions, at $H=0$ and $1 \mathrm{~T}$.

varies by less than $10 \%$ with $x$, as would be expected from the saturation moment discussed above. The value of $\theta_{\mathrm{W}}$ is positive (ferromagnetic) for $x=0$ (ref. 3), but decreases with increasing $x$ as shown in Fig. 2. In fact, $\theta_{\mathrm{W}}$ changes sign between $x=0.3$ and 0.4 , indicating that the predominant spin-spin interactions become antiferromagnetic for larger values of $x$. This could imply either that the alteration of the lattice is changing the exchange and dipole interactions between the Ho spins on the $\mathrm{R}$ sites, or that the additional spins on the $\mathrm{M}$ sites have antiferromagnetic interactions with each other or with the spins on the $\mathrm{R}$ sites.

We plot the measured specific-heat data, $C(T)$, in Fig. 3a, and show the fits used to subtract the lattice contribution. These fits were obtained from scaling polynomial fits to heatcapacity measurements on the structurally similar non-magnetic materials $\mathrm{Lu}_{2}\left(\mathrm{Ti}_{2-x} \mathrm{Lu}_{x}\right) \mathrm{O}_{7-x / 2}$, with $x=0,0.3$, and 0.67 . The magnetic specific heat was then obtained by subtracting the lattice contribution and the low-temperature Schottky peak associated with hyperfine contributions, using the fit obtained by previous workers $^{16}$. The magnetic entropy, $S(T)$, at $H=0$ and $1 \mathrm{~T}$ was then determined by integrating $C_{\text {magnetic }}(T) / T$, as shown in Fig. $3 \mathrm{c}$. This plot assumes that $S=0$ at $T=0$, and the data would extrapolate to $S(T)=R \ln 2$ at high temperatures for a system of Ising spins which explored all possible states. For $x=0$ and $H=0$, we obtained an integrated entropy at high temperatures close to $S=R[\ln 2-1 / 2 \ln (3 / 2)]$, in excellent agreement with previous work $^{16,26}$. Remarkably, despite the increased connectivity and the changing spin-spin interactions (as indicated by the changing $\theta_{\mathrm{W}}$ ), the integrated magnetic entropy at $H=0$ is essentially constant with increasing $x$. The reproducibility of the integrated entropy was within $\pm 5 \%$ from run to run, and we estimate the total uncertainty to be larger $( \pm 10 \%)$ due to uncertainty in the sample mass and the subtraction of the nuclear spin contribution to the specific heat. Although it is possible that a long-range ordering at lower temperatures (perhaps associated with the additional constraints due to stuffing of the lattice) could account for the missing entropy ${ }^{27,28}$, we see no indication of such ordering in any of our samples. Preliminary measurements of the a.c. susceptibility down to $T=60 \mathrm{mK}$ for $x=0.67$ also give no indication of such ordering (B. G. Ueland, private communication) (although if single crystals eventually become available, in-field studies could reveal interesting ordering phenomena as has been observed in the pure spin-ice materials $\mathrm{s}^{29,30}$ ). The presence of a zero-point entropy in other Ising systems is theoretically expected ${ }^{6}$, but it is striking that the same amount of zero-point entropy is retained with the increased connectivity associated with stuffing. The application of a magnetic field restored some of the 'missing' entropy for $x=0$ as first reported by Ramirez et $a l^{4}{ }^{4}$, an effect that is attributed to the decrease in the ground-state degeneracy when the system's symmetry is broken by the applied field. Because of the uncertainties in subtracting the nuclear contribution to the entropy in an applied field, the fact that the data in Fig. 3 b shows that some of the missing entropy is recovered in an applied magnetic field for normal spin ice, but is not recovered in stuffed spin-ice, should be regarded as preliminary.

The temperature dependence of the a.c. susceptibility $\left(\chi_{\text {a.c. }}\right)$ is shown in Fig. 4a, which clearly shows that frequency dependence develops below $T=3 \mathrm{~K}$ in the $x=0$ material. This is consistent with the susceptibility peak seen in previous reports on spinice materials and associated with dynamic freezing to the lowtemperature state ${ }^{17,31}$. The magnitude of the susceptibility at the lowest temperatures decreases with increasing $x$, which can be attributed to the change in $\theta_{\mathrm{W}}$, but there is also a decrease in the frequency dependence of $\chi_{\text {a.c. }}$, as is illustrated in Fig. $4 \mathrm{~b}$. The $\chi_{\text {a.c. }}(f)$ data show a monotonic decrease with increasing frequency for all of the samples, as is expected for dynamic spin freezing, but the decrease in $\chi_{\text {a.c. }}(f)$ becomes smaller and occurs at higher frequency for larger values of $x$. This trend with increasing Ho content suggests that the spin-relaxation time is shorter for larger $x$, that is, that the spins are relaxing more quickly at $T=2 \mathrm{~K}$. This is surprising because stuffing increases both the number of 

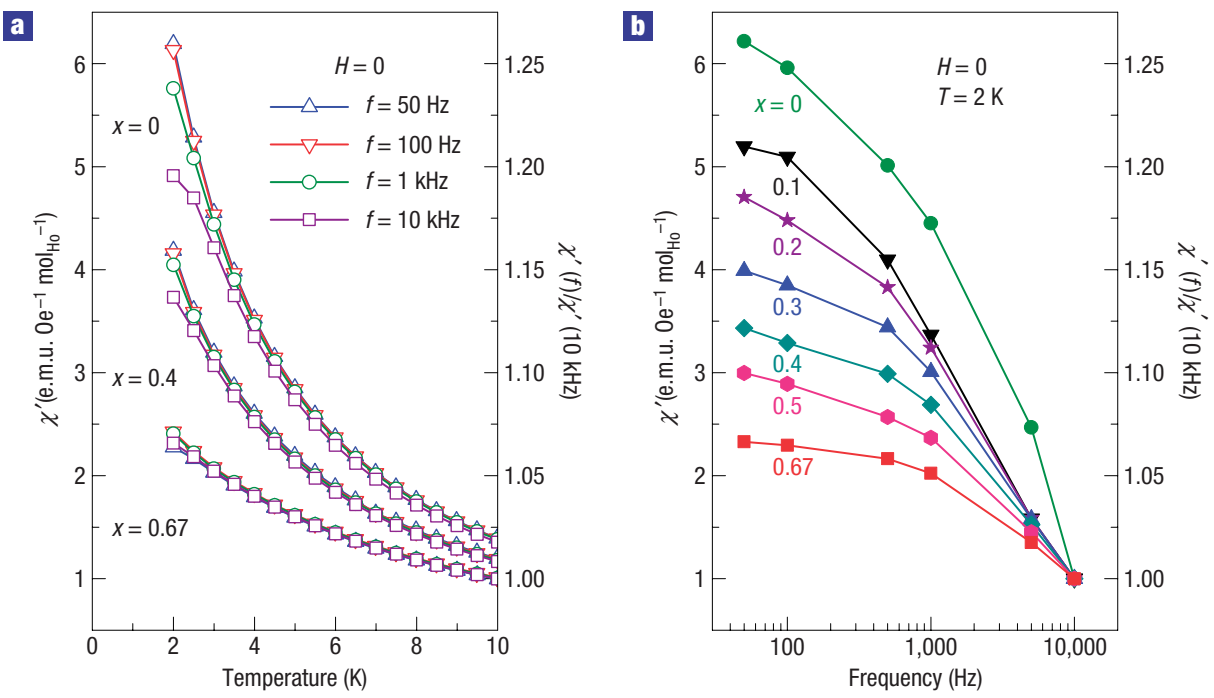

Figure 4 The low-temperature a.c. magnetic susceptibility. a, The $H=0$ a.c. susceptibility of $\mathrm{Ho}_{2}\left(\mathrm{Ti}_{2-x} \mathrm{Ho}_{x}\right) \mathrm{O}_{7-x / 2}$, for three values of $x$. The frequency-dependent peak around $T=2 \mathrm{~K}$, usually associated with dynamic freezing into a disordered state governed by the ice rules, was seen to diminish with increasing $x$. $\mathbf{b}$, The frequency dependence of the a.c. susceptibility at $T=2 \mathrm{~K}$ and $H=0$ spanning the range of $x$ studied (data are normalized to the $f=10 \mathrm{kHz}$ values).

nearest neighbours and the average spin-spin interactions, and non-magnetic dilution of other geometrically frustrated magnets, which is effectively the opposite of stuffing, actually suppresses spin freezing ${ }^{32}$.

The above results are striking, because stuffing transforms the magnetic lattice from a well ordered system of corner-sharing tetrahedra, to a disordered system of side-sharing tetrahedra, and also causes a switch from predominantly ferromagnetic to predominantly antiferromagnetic interactions. The data provide the first experimental evidence that the zero-point entropy is a feature of a broader range of frustrated Ising spin systems. That the zero-point entropy retains close to the same value in the stuffed systems may be evidence that the 'ice rules' have relevance beyond the pure spin-ice system. Perhaps more importantly, the successful synthesis of this system paves the way for new studies of all of the geometrically frustrated pyrochlore compounds as a function of increased connectivity, and thus it opens a new window on arguably the most important lattice for the study of geometrical magnetic frustration.

\section{METHODS}

Polycrystalline samples of $\mathrm{Ho}_{2}\left(\mathrm{Ti}_{2-x} \mathrm{Ho}_{x}\right) \mathrm{O}_{7-x / 2}$ were synthesized by heating the stoichiometric amounts of $\mathrm{Ho}_{2} \mathrm{O}_{3}$ (Cerac, 99.9\%) and $\mathrm{TiO}_{2}$ (Cerac, 99.9\%) at $1,700{ }^{\circ} \mathrm{C}$ in a static argon atmosphere for $12 \mathrm{~h}$. The powders were thoroughly mixed, pressed into pellets and wrapped in molybdenum foil before heating. The argon atmosphere was achieved in a vacuum furnace first evacuated to about $10^{-6}$ torr and then back-filled with argon (Airgas, 99.9\%) to room pressure.

The crystal structures were characterized through X-ray powder diffraction data using $\mathrm{Cu} \mathrm{K} \alpha$ radiation and a diffracted beam monochromator. Structural refinements were made using the Bruker AXS software package TOPAS 2.1 operated with a Pseudo-Voight TCHZ fitting profile. Ho and Ti occupancies were allowed to refine freely on both the $\mathrm{R}$ and $\mathrm{M}$ sites of the pyrochlore, with the constraint that their total occupancies from both sites maintained the correct stoichiometric ratios. The small non-zero Ti occupancy on the $\mathrm{R}$ site for stoichiometric $\mathrm{Ho}_{2} \mathrm{Ti}_{2} \mathrm{O}_{7}$ and neighbouring compositions is taken as zero within experimental uncertainty, with the $\mathrm{Ho} / \mathrm{Ti}$ mixing on the $\mathrm{R}$ site starting at $x=0.4$.
Magnetic and specific-heat measurements were carried out on pressed pellets in Quantum Design PPMS and MPMS cryostats. Heat capacity measurements used a standard semi-adiabatic heat pulse technique, and the addendum heat capacity was measured separately and subtracted. The a.c. susceptibility was measured using an a.c. excitation field of 0.5 Oe. All of the samples for susceptibility were cut to needle-like shapes, and were oriented along the direction of the applied field(s) to minimize demagnetization effects.

Received 19 January 2006; accepted 28 February 2006; published 1 April 2006.

References

1. Bernal, J. D. \& Fowler, R. H. A theory of water and ionic solution, with particular reference to hydrogen and hydroxyl ions. J. Chem. Phys. 1, 515-548 (1933).

2. Pauling, $L$. The structure and entropy of ice and of other crystals with some randomness of atomic arrangement. J. Am. Chem. Soc. 57, 2680-2684 (1935)

3. Harris, M. J., Bramwell, S. T., McMorrow, D. F., Zeiske, T. \& Godfrey, K. W. Geometrical frustration in the ferromagnetic pyrochlore $\mathrm{Ho}_{2} \mathrm{Ti}_{2} \mathrm{O}_{7}$. Phys. Rev. Lett. 79, 2554-2557 (1997).

4. Ramirez, A. P., Hayashi, A., Cava, R. J. \& Siddharthan, R. Zero-point entropy in 'spin ice'. Nature 399, 333-335 (1999).

5. Bramwell, S. T. \& Gingras, M. J. P. Spin ice state in frustrated magnetic pyrochlore materials. Science 294, 1495-1501 (2001)

6. Bramwell, S. T., Gingras, M. J. P. \& Holdsworth, P. C. W. in Frustrated Spin Systems (ed. Diep, H. T.) $367-456$ (World Scientific, Singapore, 2004).

7. Higashinaka, R., Fukazawa, H. \& Maeno, Y. Anisotropic release of the residual zero-point entropy in the spin ice compound $\mathrm{Dy}_{2} \mathrm{Ti}_{2} \mathrm{O}_{7}$ : Kagome ice behaviour. Phys. Rev. B 68, 014415 (2003).

8. Ramirez, A. P. in Handbook of Magnetic Materials Vol. 13 (ed. Buschow, K. J. H.) 423-520 (Elsevier Science, Amsterdam, 2001).

9. Moessner, R. Magnets with strong geometric frustration. Can. J. Phys. 79, 1283-1294 (2001).

10. Gaulin, B. D. \& Gardner, J. S. in Frustrated Spin Systems (ed. Diep, H. T.) 457-490 (World Scientific, Singapore, 2004).

11. Raju, N. P., Dion, M., Gingras, M. J. P., Mason, T. E. \& Greedan, J. E. Transition to long-range magnetic order in the highly frustrated insulating pyrochlore antiferromagnet $\mathrm{Gd}_{2} \mathrm{Ti}_{2} \mathrm{O}_{7}$. Phys. Rev. $B$ 59, 14489-14498 (1999).

12. Ramirez, A. P. et al. Multiple field-induced phase transitions in the geometrically frustrated dipolar magnet: $\mathrm{Gd}_{2} \mathrm{Ti}_{2} \mathrm{O}_{7}$. Phys. Rev. Lett. 89, 067202 (2002).

13. Champion, J. D. M. et al. $\mathrm{Er}_{2} \mathrm{Ti}_{2} \mathrm{O}_{7}$ : evidence of quantum order by disorder in a frustrated antiferromagnet. Phys. Rev. B 68, 020401 (2003).

14. Hodges, J. A. et al. First-order transition in the spin dynamics of geometrically frustrated $\mathrm{Yb}_{2} \mathrm{Ti}_{2} \mathrm{O}_{7}$ Phys. Rev. Lett. 88, 077204 (2002).

15. Gardner, J. S. et al. Cooperative paramagnetism in the geometrically frustrated pyrochlore antiferromagnet $\mathrm{Tb}_{2} \mathrm{Ti}_{2} \mathrm{O}_{7}$. Phys. Rev. Lett. 82, 1012-1015 (1999).

16. Bramwell, S. T. et al. Spin correlations in $\mathrm{Ho}_{2} \mathrm{Ti}_{2} \mathrm{O}_{7}$ : a dipolar spin ice system. Phys. Rev. Lett. 87, 047205 (2001).

17. Matsuhira, K., Hinatsu, Y., Tenya, K. \& Sakakibara, T. Low temperature magnetic properties of frustrated pyrochlore ferromagnets $\mathrm{Ho}_{2} \mathrm{Sn}_{2} \mathrm{O}_{7}$ and $\mathrm{Ho}_{2} \mathrm{Ti}_{2} \mathrm{O}_{7}$. J. Phys. Condens. Matter 12, L649-L656 (2000).

18. Fukazawa, H., Melko, R. G., Higashinaka, R., Maeno, Y. \& Gingras, M. J. P. Magnetic anisotropy of the spin-ice compound $\mathrm{Dy}_{2} \mathrm{Ti}_{2} \mathrm{O}_{7}$. Phys. Rev. B 65, 054410 (2002).

19. Snyder, J. et al. Low-temperature spin freezing in the $\mathrm{Dy}_{2} \mathrm{Ti}_{2} \mathrm{O}_{7}$ spin ice. Phys. Rev. B 69, 064414 (2004).

20. Wiebe, C. R. et al. Frustration-driven spin freezing in the $\mathrm{S}=1 / 2$ fcc perovskite $\mathrm{Sr}_{2} \mathrm{MgReO}_{6}$. Phys. Rev. B 68, 134410 (2003). 
21. Karunadasa, H., Huang, Q., Ueland, B. G., Schiffer, P. \& Cava, R. J. $\mathrm{Ba}_{2} \mathrm{LnSbO}_{6}$ and $\mathrm{Sr}_{2} \mathrm{LnSbO}_{6}$ ( $\mathrm{Ln}=\mathrm{Dy}, \mathrm{Ho}, \mathrm{Gd})$ double perovskites: lanthanides in the geometrically frustrating fcc lattice. Proc. Natl Acad. Sci. USA 100, 8097-8102 (2003).

22. Subramanian, M. A., Aravamudan, G. \& Rao, G. V. S. Oxide pyrochlores-a review. Prog. Solid State Chem. 15, 55-143 (1983).

23. Sukhanova, G. E., Guseva, K. N., Kolesnikov, A. V. \& Shcherbakova, L. G. Phase-equilibria in the $\mathrm{TiO}_{2}-\mathrm{Ho}_{2} \mathrm{O}_{3}$ system. Inorg. Mater. 18, 1742-1745 (1982).

24. Shamrai, G. V., Magunov, R. L., Sadkovskaya, L. V., Stasenko, I. V. \& Kovalevskaya, I. P. The system $\mathrm{Ho}_{2} \mathrm{O}_{3}-\mathrm{TiO}_{2}$. Inorg. Mater. 27, 140-141 (1991).

25. Snyder, J. Thesis, Pennsylvania State Univ. (2003).

26. Cornelius, A. L. \& Gardner, J. S. Short-range magnetic interactions in the spin-ice compound $\mathrm{Ho}_{2} \mathrm{Ti}_{2} \mathrm{O}_{7}$. Phys. Rev. B 64, 060406 (2001).

27. Melko, R. G., den Hertog, B. C. \& Gingras, M. J. P. Long-range order at low temperatures in dipolar spin ice. Phys. Rev. Lett. 87, 067203 (2001).

28. Siddharthan, R., Shastry, B. S. \& Ramirez, A. P. Spin ordering and partial ordering in holmium titanate and related systems. Phys. Rev. B 63, 184412 (2001).

29. Higashinaka, R. \& Maeno, Y. Field-induced transition on a triangular plane in the spin-ice compound $\mathrm{Dy}_{2} \mathrm{Ti}_{2} \mathrm{O}_{7}$. Phys. Rev. Lett. 95, 237208 (2005).
30. Ruff, J. P. C., Melko, R. G. \& Gingras, M. J. P. Finite-temperature transitions in dipolar spin ice in a large magnetic field. Phys. Rev. Lett. 95, 097202 (2005).

31. Matsuhira, K., Hinatsu, Y. \& Sakakibara, T. Novel dynamical magnetic properties in the spin ice compound $\mathrm{Dy}_{2} \mathrm{Ti}_{2} \mathrm{O}_{7}$. J. Phys. Condens. Matter 13, L737-L746 (2001).

32. Ramirez, A. P., Espinosa, G. P. \& Cooper, A. S. Strong frustration and dilution-enhanced order in a quasi-2D spin glass. Phys. Rev. Lett. 64, 2070-2073 (1990).

\section{Acknowledgements}

The authors gratefully acknowledge financial support from the National Science Foundation and helpful discussions with R. Moessner and A. P. Ramirez. R.S.F. thanks the CNPq-Brazil for sponsorship.

Correspondence and requests for materials should be addressed to R.J.C. or P.S.

\section{Competing financial interests}

The authors declare that they have no competing financial interests.

Reprints and permission information is available online at http://npg.nature.com/reprintsandpermissions/ 